\title{
INFLUÊNCIA DE SISTEMAS DE PRODUÇÃO SOBRE A OCORRÊNCIA DE INIMIGOS NATURAIS DE AFÍDEOS EM POMARES DE PESSEGUEIROS EM ARAUCÁRIA-PR ${ }^{1}$
}

\author{
JOSELIAMARIA SCHUBER ${ }^{2}$,LINO BITTENCOURT MONTEIRO ${ }^{3}$, ALEX SANDRO POLTRONIERI ${ }^{2}$, \\ NERIO APARECIDO CARDOSO ${ }^{2}$, LOUISE LARISSA MAY DE MIO
}

RESUMO - A pressão da sociedade tem forçado a adoção de sistemas de produção de frutas mais sustentáveis e de menor impacto ambiental. O objetivo deste trabalho foi verificar a influência dos sistemas de produção de Boas Práticas Agrícolas (BPA) e Convencional (PC) sobre a diversidade de inimigos naturais de afídeos presentes em seis pomares de pessegueiros no município de Araucária-PR, de julho de 2005 a setembro de 2006. O levantamento do número de inimigos naturais foi realizado por meio de cinco métodos de amostragens: visual em plantas de pessegueiros e em plantas invasoras; armadilhas Möericke; funil e adesivas. No sistema BPA, foram coletados mais espécimes de inimigos naturais (53\%), em relação aos pomares PCs (46\%), havendo a ocorrência, em ambos, de predadores das famílias Syrphidae, Coccinellidae e Chrysopidae e parasitóides da ordem Hymenoptera. Do total de inimigos naturais coletados, verificou-se a ocorrência de maior quantidade de predadores de afídeos.

Termos para indexação: Boas Práticas Agrícolas, Sistema Convencional.

\section{INFLUENCE OF SYSTEMS OF PRODUCTION ON THE OCCURRENCE OF NATURAL ENEMIES OF APHIDS ON ORCHARDS OF PEACH TREES IN ARAUCARIA, PR}

\begin{abstract}
The pressure of the society has forced the adoption of more sustainable fruit production systems and with less environmental impact. The objective of this work was to verify the influence of production systems of Good Agricultural Practices (GAP) and Conventional Production (CP), about the diversity of natural enemies of aphids present on six orchards of peach trees in the city of Araucaria, PR, from July 2005 to September 2006. The evaluation of the number of natural enemies of aphid's species was made by means of five sampling methods: visual in plants of peach trees; visual in invading plants; yellow water traps of Möericke type; funnel and adhesive traps. In the system GPA, more specimens of natural enemies had been collected (53\%) in relation to orchards CP (46\%), having the occurrence, in both orchards, of predators of the Syrphidae, Coccinellidae and Chrysopidae family and parasitoids of Hymenoptera order. Concerning the total of collected natural enemies, it was verified the greatest occurrence of aphid predators.
\end{abstract}

Index terms: Good Agricultural Practices, Conventional Production.

\section{INTRODUÇÃO}

Durante as últimas décadas, ocorreu uma intensificação do uso de insumos agrícolas para atender à demanda proveniente da adoção de Sistemas Convencionais de produção no Brasil (Campanhola et al., 1997), os quais objetivam a maximização da produção, não levando em consideração a qualidade dos frutos e a preservação ambiental (Marangoni et al., 1995).

Segundo Fadini \& Louzada (2001), a intensidade dos impactos ambientais causados pelas práticas agrícolas está diretamente relacionada com o sistema de produção utilizado. Atualmente, existe maior integração da fruticultura com recursos ambientais, como a diversidade de plantas e a presença de inimigos naturais (Marangoni et al., 1995). Um exemplo disso são as Boas Práticas Agrícolas, as quais englobam um conjunto de instruções que, quando normalizadas, levam à Produção Integrada de Frutas (PIF). A PIF é apontada como uma alternativa para a produção de frutas de qualidade, por meio de uma visão multidisciplinar e não pela aplicação de práticas isoladas, como ocorre na fruticultura convencional (Fachinello \& Herter, 2001). O objetivo deste trabalho foi verificar a influência de sistemas de produção sobre a diversidade de inimigos naturais associados com afídeos presentes em pomares de pessegueiros no município de Araucária-PR.

\footnotetext{
'(Trabalho 148-07). Recebido em: 15-06-2007. Aceito para publicação em: 10-04-2008. Parte da dissertação de mestrado do primeiro autor, PPGPV/ UFPR

${ }^{2}$ Mestrando do curso de Pós-graduação em Produção Vegetal da Universidade Federal do Paraná, Rua dos Funcionários, 1540, 80035-050 Curitiba-PR; Email: joseliaschuber@yahoo.com.br, alex.poltronieri@yahoo.com.br.e neriocardoso@hotmail.com.

${ }^{3}$ Depto. De Fitotecnia e Fitossanitarismo, SCA/UFPR. Rua dos Funcionários, 1540, 80035-050 Curitiba-PR; E-mail: lbmonteiro@terra.com.br, maydemio@ufpr.br.
} 


\section{MATERIAL E MÉTODOS}

\section{Localização e caracterização dos pomares}

$\mathrm{O}$ experimento foi realizado em Araucária-PR (latitude:

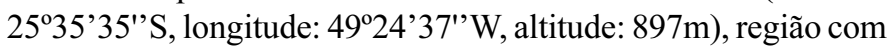
temperatura média anual de $16^{\circ} \mathrm{C}$, umidade relativa do ar de $80 \%$ e precipitação pluviométrica em torno de $1.500 \mathrm{~mm}$ por ano (SIMEPAR, 2006).

A influência de sistemas de produção sobre a presença de inimigos naturais de afídeos foi verificada em seis pomares de pessegueiros 'Chimarrita', apresentando em média 0,44 ha e 150 plantas, conduzidas em taça com quatro pernadas, espaçadas em $6 \times 4$ m e idade média aproximada de 8 anos.

Os pomares foram conduzidos em dois sistemas de produção, sendo que três deles adotavam as normas de Boas Práticas Agrícolas (BPA) (BPA1, BPA2 e BPA3) e os demais seguiram o Sistema de Produção Convencional (PC) (PC1, PC2 e PC3). Os pomares BPAs estavam em processo de adoção das Normas de Produção Integrada de Frutas (NPIF) (Fachinello \& Herter, 2001). Nestes pomares,foram utilizadas armadilhas para o monitoramento das principais pragas, o que serviu de base para as pulverizações com inseticidas. O manejo do solo permitiu uma cobertura vegetal durante todo o ano, restringindo o uso de herbicidas. Nos pomares BPA1 e BPA2, foi feito o plantio de ervas para a cobertura do solo.

As práticas culturais adotadas nos pomares PCs foram estabelecidas pelo produtor com base em sua experiência, sendo que as aplicações de produtos fitossanitários foram realizadas de acordo com um calendário fixo. Somente no pomar PC3 foram usadas armadilhas de monitoramento. $\mathrm{O}$ manejo das plantas invasoras foi realizado exclusivamente com o uso de herbicidas no pomar PC1, com grade na linha de plantio associada com herbicidas no PC2 e no pomar PC3, o solo ficou permanentemente coberto com plantas invasoras, as quais foram manejadas com roçadas e aplicações de herbicidas.

Os produtos fitossanitários utilizados para o manejo de pragas e das plantas invasoras nos pomares estudados estão apresentados no Tabela 1 .

\section{Coletas de afídeos e inimigos naturais}

As coletas foram realizadas de julho de 2005 a setembro de 2006 pelos métodos de amostragens: visual em pessegueiros, visual em plantas invasoras, armadilhas do tipo Möericke, funil e adesivas, com o objetivo de se coletar a maior diversidade de espécies de inimigos naturais.

Os insetos capturados foram separados no Laboratório de Manejo Integrado de Pragas (UFPR),e a identificação dos gêneros e espécies foi realizada por meio de chaves, coleções de referência, artigos específicos e,posteriormente,confirmadas por especialistas. As múmias de afídeos coletadas foram colocadas em cápsulas de gelatina,individualmente,sob a temperatura de $25^{\circ} \mathrm{C} \pm 2^{\circ} \mathrm{C}$, UR de $80 \% \pm 5^{\circ} \mathrm{C}$ e fotofase de 14 horas, até a emergência dos parasitóides.

Amostragem Visual em Pessegueiros. Todos os ramos e folhas de cinco pessegueiros, escolhidos aleatoriamente e por pomar, distribuídos até $1,70 \mathrm{~m}$ de altura, foram observados visualmente. Os insetos foram coletados com auxílio de pinças, pincéis, tubos de ensaio e acondicionados em potes plásticos previamente identificados. Estes foram levados para o laboratório e armazenados em álcool 70\% para contagem e identificação.

Amostragem Visual em Plantas Invasoras. Em cada pomar,definiram-se, aleatoriamente e de maneira eqüidistante, três áreas de $24 \mathrm{~m}^{2}$ situadas nas entrelinhas. Todas as plantas contidas nessas áreas foram vistoriadas visualmente para se verificar a presença de insetos, os quais foram coletados com auxílio de pinças, pincéis, tubos de ensaio, acondicionados em potes plásticos previamente identificados e transportados ao laboratório para contagem e identificação.

Möericke. Quatro armadilhas do tipo Möericke (29 cm x $20 \mathrm{~cm}$ x $6 \mathrm{~cm}$ de altura) foram instaladas nas entrelinhas de cada pomar, a uma altura média de $90 \mathrm{~cm}$, contendo 1,25 L de água e 2 $\mathrm{mL}$ de detergente incolor neutro para quebrar a tensão superficial. Semanalmente,os insetos foram retirados com o auxílio de uma pisseta e transferidos para recipientes plásticos, sendo o líquido renovado.

Armadilhas Adesivas. Quatro placas adesivas Biotrap ${ }^{\circledR}$, Biocontrole, SP $(24 \mathrm{~cm} \times 10 \mathrm{~cm})$ de coloração amarela e azul, foram distribuídas por pomar, a 1,70 m de altura em árvores escolhidas aleatoriamente, contendo uma placa de cada cor. Mensalmente,as placas foram substituídas,e os insetos foram contados no laboratório.

Funil. O método foi preconizado pela ACTA/OILB (1974), constituindo-se de um funil metálico de $0,60 \mathrm{~m}$ de diâmetro superior, $0,10 \mathrm{~m}$ de diâmetro inferior e $0,40 \mathrm{~m}$ de altura, sendo colocado embaixo de ramos de dez pessegueiros para que os insetos caíssem no funil após três batidas em ramos secundários, com um bastão. As coletas foram realizadas semanalmente, de setembro de 2005 a setembro de 2006, com exceção de abril a julho de 2006, período em que o pessegueiro se encontrava sem folhas.

Coeficientes de alteração dos componentes dos sistemas de produção

Os sistemas de produção foram caracterizados segundo os estudos de Kovach et al. (1992) e Rodrigues et al. (2002), cujos componentes dos sistemas foram agrupados em três classes: manejo de pragas, manejo de plantas invasoras e inimigos naturais. Para cada componente,foi atribuído um coeficiente de alteração (CA) proposto por Rodrigues et al. (2002), cujos valores são: (+3), (+1), (-1) e (-3) (Tabela 2).

Manejo de pragas. Os fatores que definem a classe manejo de pragas foram analisados quanto ao número de aplicações de inseticidas e presença de monitoramento. Para a definição do CA do componente aplicação de inseticidas, considerou-se que, para o controle de Grapholita molesta (Lepidoptera: Tortricidae) e Anastrepha sp. (Diptera: Tephritidae),é necessário em Araucária uma aplicação de inseticida para cada praga em 'Chimarrita', baseado em trabalhos realizados pelo Grupo de Ensino, Extensão e Pesquisa em Produção Integrada (GEPPI) e informações disponibilizadas pela EMATER-PR.

Para a definição do CA do componente presença de 
monitoramento,considerou-se que o uso de armadilhas é essencial para o manejo de G. molesta e Anastrepha sp., assim o monitoramento de apenas uma dessas espécies foi considerado como um aspecto negativo para a tomada de decisão de controle, sendo ideal o monitoramento das duas espécies.

Manejo de plantas invasoras. Os fatores levados em consideração foram os números de aplicações de herbicidas, tipo de manejo, diversidade e distribuição de plantas invasoras. O CA do número de aplicações de herbicidas foi realizado com base NPIF e Titi et al. (1995). Definiu-se como medida ideal a nãointervenção de herbicidas nos pomares,e o seu uso,um aspecto negativo.

O CA do tipo de manejo de plantas invasoras baseou-se nas NPIF que considera como manejo ideal o uso de capina manual e mecânica, mulching, plantio de cobertura, entre outros. A ausência de um ou mais fatores é considerado como um aspecto negativo.

A análise da diversidade das plantas invasoras foi baseada no número máximo de espécies encontradas nos seis pomares,relacionando-as ao número de espécies encontradas em cada um dos pomares. Considerou-se que o maior número de espécies tende a promover maior equilíbrio da fauna.

O CA da distribuição de plantas invasoras foi considerado a presença de espécies de invasoras na linha e entrelinha do plantio,e que a manutenção das mesmas é um aspecto positivo, sendo um fator negativo a redução da área com plantas invasoras.

Inimigos naturais. Os fatores basearam-se na diversidade, densidade e seletividade de inseticidas aos inimigos naturais. A diversidade foi uma relação entre o número total de espécies coletadas em todos os pomares e o total de espécies coletadas em cada um dos pomares. Os coeficientes de densidade foram definidos a partir da relação entre o número total de espécimes capturados e o número de espécimes capturados em cada um dos pomares, sendo positivo o pomar que apresentar o maior valor de diversidade e densidade. A seletividade dos inseticidas foi definida com base nos estudos: SPV (1983); Union Internacionale des Sciences Biologiques (1986); Agnello et al. (1993); ACTA (1993); Hull et al. (1994); Delabays et al. (2003a); Delabays et al. (2003b) e ACTA (2006)

Aos CAs obtidos definiram cinco graus de impacto ambiental, tendo valor máximo de 54 e mínimo de -54: Grande diminuição da possibilidade de impacto ambiental (GD) (33 a 54), Moderada diminuição da possibilidade de impacto ambiental (MD) (10 a 32), Componentes inalterados quanto ao impacto ambiental (CI) (-12 a 9), Moderado aumento da possibilidade de impacto ambiental (MA) (-32 a -11) e Grande aumento da possibilidade de impacto ambiental (GA) (-33 a -54).

\section{Análise estatística}

As análises das variáveis dependentes para comparar métodos de amostragem foram realizadas por meio da análise de variância em delineamento completamente casualizado e, para atender aos pressupostos de normalidade e homogeneidade, os dados foram transformados ( $\log \mathrm{X})$. A comparação entre as variáveis dependentes foi realizada por meio do teste de comparação múltipla de Tukey 5\% (Barbin, 2004). Por meio do teste de Correlação de Pearson, foi analisada a relação entre as variáveis, inimigos naturais e afídeos.

\section{RESULTADOS E DISCUSSÃO}

Os componentes que caracterizam os pomares em estudo foram apresentados na Tabela 2,que serviu de base para definir os coeficientes de alterações totais e os respectivos conceitos em cada pomar (Tabela 3 ).

A análise dos componentes das classes (Manejo de pragas, Manejo de plantas invasoras e Inimigos naturais) mostrou que o Sistema de Boas Práticas Agrícolas (BPA) foi o que obteve os menores impactos ambientais (Tabela 3 ). Segundo Rodrigues et al. (2002), as tecnologias agropecuárias devem ser recomendadas e adotadas com segurança após a avaliação sistemática dos impactos.

Dentre os pomares de Produção Convencional (PC),verificou-se que o PC1 e PC2 foram os considerados causadores de maior impacto ambiental, devido à aplicação excessiva de inseticidas e herbicidas, à exposição do solo às intempéries durante boa parte do ano, o que reduz a diversidade de plantas invasoras e influenciando negativamente a entomofauna benéfica.

O controle de Grapholita molesta e de Anastrepha sp., na Produção Convencional (PC), foi realizado com 50\% mais inseticidas em relação ao Sistema de Boas Práticas Agrícolas (BPA) (Tabela 1). O menor número de aplicações de inseticidas nos pomares BPAs pode ser explicado pela adoção do monitoramento de pragas, o qual fornece subsídios para a correta aplicação (Pineda, 2005), influenciando na abundância de organismos benéficos e reduzindo o impacto ambiental (Altieri et al., 2003). No segundo ano, houve uma redução de $57 \%$ no uso de inseticidas em ambos os sistemas, em função da diminuição das atividades fitossanitárias, devido às perdas na produção, causadas pelo abortamento das flores em função das baixas temperaturas e geadas verificadas entre agosto e setembro de 2006.

$\mathrm{Na}$ avaliação da diversidade de espécies de plantas invasoras,observou-se que os pomares BPAs apresentaram maior número em relação aos pomares PCs, sendo coletadas,respectivamente, um total de 20 e 12 espécies, nos dois anos (Tabela 4). O resultado encontrado nos pomares PCs deve-se provavelmente ao uso freqüente de herbicidas, que podem causar mudanças nas populações de invasoras e/ou selecionar biótipos resistentes em detrimento de membros suscetíveis da comunidade (Altieri et al., 2003).

Pode-se verificar que os pomares BPA3 e PC3, apesar de serem enquadrados em diferentes sistemas, receberam o mesmo conceito quanto ao impacto ambiental (CI) (Tabela 3), mostrando que é possível melhorar o sistema convencional quando se adota o monitoramento de pragas e se faz a manutenção da cobertura do solo.

Observou-se que os coeficientes de alteração evoluíram positivamente de 2005/06 para 2006/07, principalmente nos 
pomares BPA2 e nos pomares PCs (Tabela 3). A redução de inseticidas, em torno de $50 \%$ no segundo ano, proporcionou melhora nos fatores avaliados no Sistema Convencional, o que mostra a necessidade de rever as pulverizações seqüenciais e priorizar a utilização de produtos fitossanitários que sejam eficientes para o manejo de pragas, entretanto sem causar prejuízos à entomofauna e ao meio ambiente (Fachinello \& Herter 2001; Fachinello et al., 2003).

O número de insetos capturados foi de 1.296 predadores e parasitóides pertencentes às famílias Syrphidae, Coccinellidae e Chrysopidae, e à ordem Hymenoptera (Tabela 5), correspondendo, respectivamente, a $58 \%$ de predadores e $42 \%$ de parasitóides.

Não houve diferença significativa $(\mathrm{p}<0,05)$ entre os números predadores nos pomares. Observou-se que, em dois pomares BPAs,as capturas foram superiores a $20 \%$, enquanto, isso ocorreu em apenas um pomar no PC (Tabela 5).

No presente estudo,observou-se no pomar PC2 as mais baixas capturas de coccinelídeos e sirfídeos (Tabela 5), provavelmente devido ao uso intensivo de inseticidas, herbicidas e grade, que, além do impacto direto sobre inimigos naturais, reduziu a população de plantas invasoras, limitando a presença dos mesmos (Price, 1980). Áreas com diversidade de plantas invasoras apresentam um micro-habitat favorável para o desenvolvimento de insetos entomófagos generalistas, devido à ocorrência de presas alternativas, pólen e néctar (Altieri et al., 2005). Isso pode ser verificado nos pomares BPAs (Figura 1), onde se observa a tendência de maior número de coccinelídeos e sirfídeos entre os meses de agosto e novembro, correspondendo à fase de florescimento das plantas invasoras em Araucária (Altieri et al., 2003).
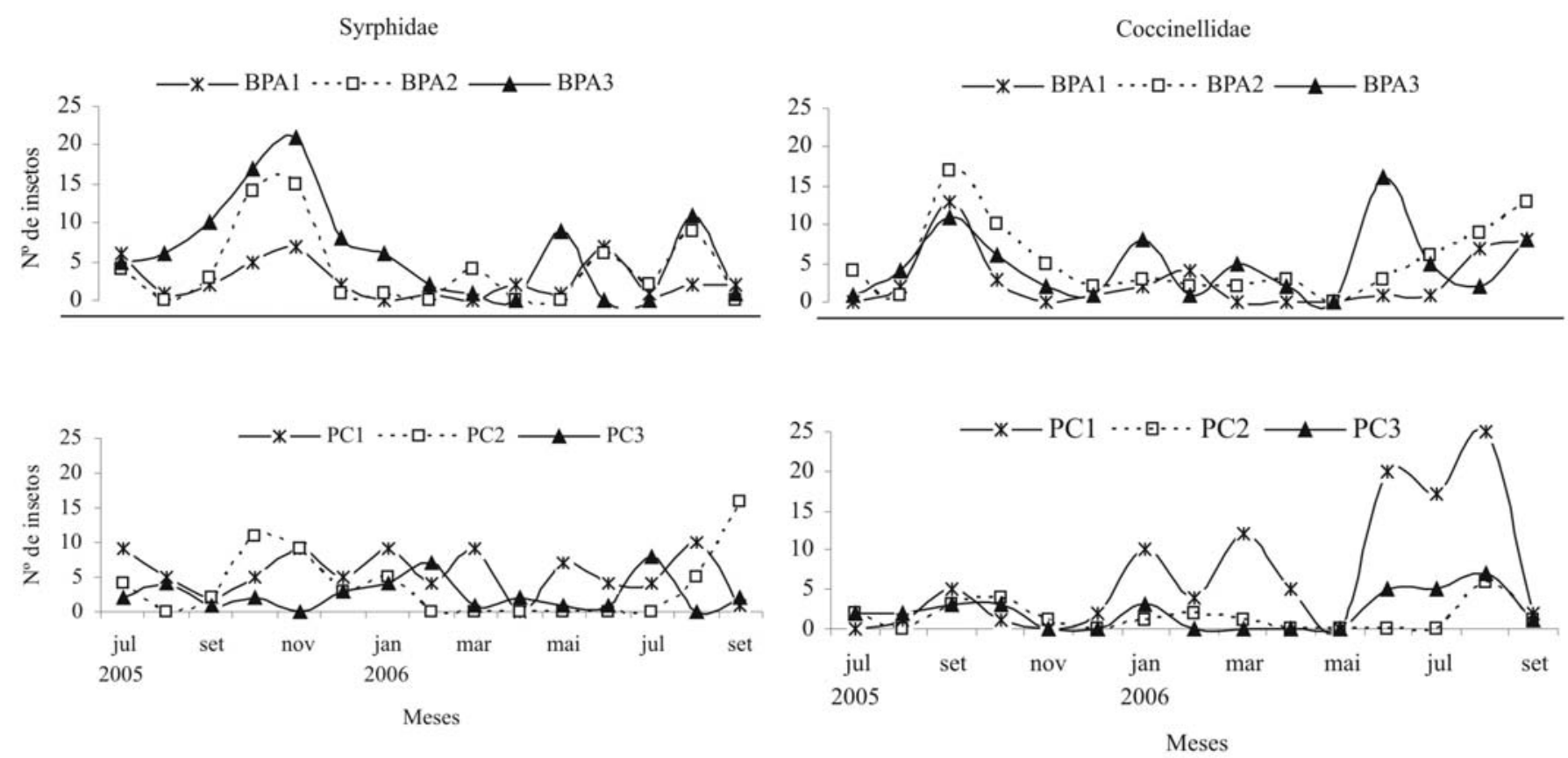

FIGURA 1 - Flutuação populacional de coccinelídeos e sirfídeos em seis pomares de pessegueiros conduzidos pelos sistemas de Boas Práticas Agrícolas (BPA) e Convencional (PC). Araucária-PR. Julho de 2005 a setembro de 2006. 


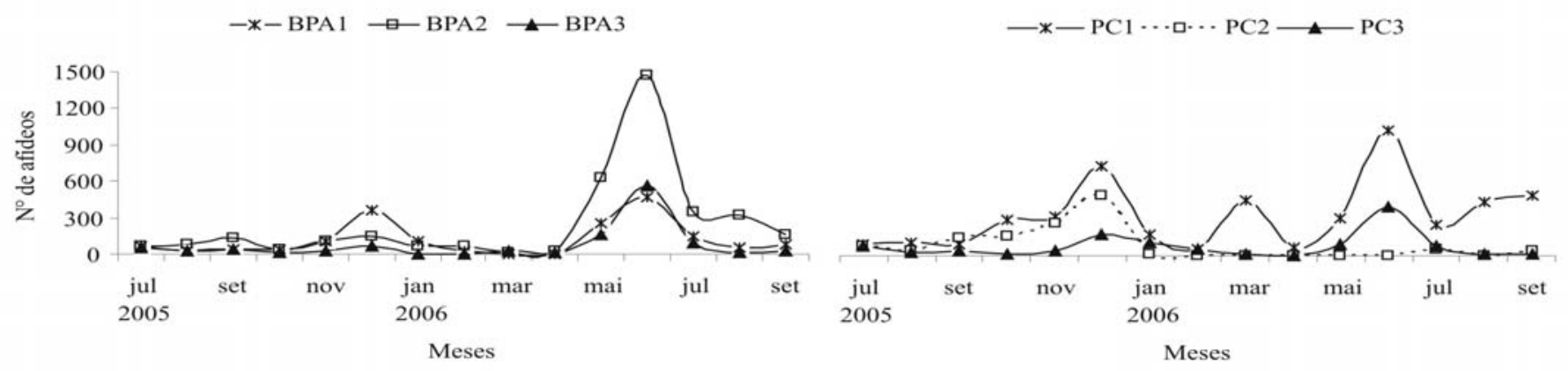

FIGURA 2 - Flutuação populacional de afídeos em seis pomares de pessegueiros conduzidos pelos sistemas de Boas Práticas Agrícolas (BPA) e Convencional (PC). Araucária-PR. Julho de 2005 a setembro de 2006.

TABELA 1 - Inseticidas e manejos de plantas invasoras realizados em pomares de pessegueiros 'Chimarrita' conduzidos nos sistemas de Boas Práticas Agrícolas (BPA) e Convencional (PC) em Araucária-PR. 2005/06 e 2006/07.

\begin{tabular}{|c|c|c|c|c|}
\hline \multirow{2}{*}{$\begin{array}{l}\text { Sistemas de } \\
\text { produção }\end{array}$} & \multicolumn{2}{|c|}{ Ciclo vegetativo $2005 / 06$} & \multicolumn{2}{|c|}{ Ciclo vegetativo $2006 / 07$} \\
\hline & Mês & Manejo fitossanitário & Mês & Manejo fitossanitário \\
\hline BPA1 & $\begin{array}{l}\text { novembro } \\
\text { dezembro }\end{array}$ & $\begin{array}{l}\text { roçada mecânica } \\
\text { deltamethrina }\end{array}$ & $\begin{array}{l}\text { janeiro } \\
\text { julho } \\
\text { agosto }\end{array}$ & $\begin{array}{l}\text { roçada mecânica } \\
\text { roçada mecânica } \\
\text { fenthion }\end{array}$ \\
\hline \multirow[t]{3}{*}{ BPA2 } & outubro & fenthion & março & rolo-faca \\
\hline & novembro & fenthion, dimethoate & maio & herbicida \\
\hline & dezembro & herbicida & outubro & fenthion \\
\hline \multirow[t]{6}{*}{ BPA3 } & outubro & fenitrothion & janeiro & roçada mecânica \\
\hline & outubro & herbicida & abril & roçada mecânica \\
\hline & novembro & triflumurom & junho & roçada manual \\
\hline & dezembro & fenitrothion & agosto & herbicida, roçada mecânica \\
\hline & dezembro & $2 x$ roçada manual & outubro & $2 \mathrm{x}$ dimethoate \\
\hline & & & $\begin{array}{l}\text { outubro } \\
\text { dezembro }\end{array}$ & $\begin{array}{l}\text { herbicida, roçada mecânica } \\
2 x \text { rocada manual }\end{array}$ \\
\hline \multirow[t]{4}{*}{$\mathrm{PC} 1$} & setembro & $3 \times$ Fenthion & março & herbicida \\
\hline & outubro & $2 \mathrm{x}$ Fenthion & agosto & herbicida \\
\hline & outubro & herbicida & setembro & $2 \mathrm{x}$ fenthion \\
\hline & $\begin{array}{l}\text { novembro } \\
\text { dezembro }\end{array}$ & herbicida & $\begin{array}{l}\text { outubro } \\
\text { outubro }\end{array}$ & $\begin{array}{l}\text { fenthion } \\
\text { herbicida }\end{array}$ \\
\hline \multirow{7}{*}{$\mathrm{PC} 2$} & setembro & $\operatorname{grade}^{1}$ & julho & grade \\
\hline & outubro & $2 \mathrm{x}$ fenthion & setembro & fenthion \\
\hline & outubro & herbicida & setembro & grade \\
\hline & novembro & fenthion, dimethoate & outubro & grade, herbicida \\
\hline & novembro & herbicida & novembro & lambdacyhalothrin \\
\hline & dezembro & grade & & deltamethrina \\
\hline & dezembro & dimethoate & & \\
\hline \multirow[t]{5}{*}{$\mathrm{PC} 3$} & outubro & fenitrothion & agosto & herbicida \\
\hline & outubro & herbicida & outubro & roçada mecânica \\
\hline & novembro & fenitrothion & outubro & $2 \mathrm{x}$ dimethoate \\
\hline & dezembro & fenitrothion & dezembro & dimethoate \\
\hline & dezembro & roçada mecânica & dezembro & roçada mecânica \\
\hline
\end{tabular}

${ }^{1}$ Utilizou-se grade, acoplada a um trator, para o controle de plantas invasoras.

TABELA 2 - Fatores que caracterizam os sistemas de Boas Práticas Agrícolas (BPA) e Produção Convencional (PC), em pomares de pessegueiros 'Chimarrita'. Araucária-PR. Julho de 2005 a setembro de 2006.

\begin{tabular}{|c|c|c|c|c|c|}
\hline \multirow{2}{*}{ Classes } & \multirow{2}{*}{ Componentes } & \multicolumn{4}{|c|}{ Coeficientes de Alteração / Fatores dos componentes } \\
\hline & & 3 & 1 & -1 & -3 \\
\hline \multirow[t]{2}{*}{ Manejo de pragas } & Número de inseticida & 1 & 2 & 3 & $>3$ \\
\hline & Monitoramento & $\begin{array}{l}\text { Grapholita molesta } \mathrm{e} \\
\text { Anastrepha } \mathrm{sp} .\end{array}$ & $\begin{array}{c}\text { Grapholita molesta } \\
\text { ou Anastrepha } \mathrm{sp} .\end{array}$ & $-(1)$ & $\begin{array}{c}\text { sem } \\
\text { monitoramento }\end{array}$ \\
\hline \multirow{4}{*}{$\begin{array}{l}\text { Manejo de plantas } \\
\text { invasoras }\end{array}$} & Número de herbicida & 0 & 1 & 2 & $>2$ \\
\hline & Tipo de manejo & $\begin{array}{l}\text { vegetado, mulching e } \\
\text { plantio de cobertura }\end{array}$ & $\begin{array}{l}\text { vegetado e capina } \\
\text { manual }\end{array}$ & $\begin{array}{l}\text { vegetado e capina } \\
\text { mecânica }\end{array}$ & $\begin{array}{l}\text { grade, sem plantio } \\
\text { de cobertura }\end{array}$ \\
\hline & Diversidade de espécie & $>10$ & 6 a 9 & 2 a 5 & 0 a 1 \\
\hline & Distribuição pl. invasoras & linha e entrelinha & entrelinha & linha & ausência \\
\hline \multirow[t]{2}{*}{ Inimigos naturais } & Diversidade de espécie & 13 a 16 & 9 a 12 & 5 a 8 & 0 a 4 \\
\hline & $\begin{array}{l}\text { Densidade } \\
\text { Seletividade de inseticida }\end{array}$ & $\begin{array}{c}>15 \% \text { do total } \\
\text { neutro }\end{array}$ & $\begin{array}{l}11 \text { a } 14 \% \text { do total } \\
\text { pouco tóxico }\end{array}$ & $\begin{array}{c}7 \text { a } 10 \% \text { do total } \\
\text { tóxico }\end{array}$ & $\begin{array}{l}3 \text { a } 6 \% \text { do total } \\
\text { muito tóxico }\end{array}$ \\
\hline
\end{tabular}

(1) Não se considerou nenhum fator para o componente monitoramento, para o coeficiente de alteração -1 . 
TABELA 3 - Coeficientes de alteração dos componentes (CA) e conceitos de impacto ambiental dos sistemas de Boas Práticas Agrícolas (BPA) e Produção Convencional (PC) em pomares de pessegueiros. Araucária-PR. Ciclo vegetativo 2005/2006 e 2006/ 2007.

\begin{tabular}{|c|c|c|c|c|c|c|c|c|}
\hline \multirow{2}{*}{$\begin{array}{c}\text { Ciclo } \\
\text { vegetativo }\end{array}$} & \multirow{2}{*}{ Classes } & \multirow{2}{*}{ Componentes } & \multicolumn{6}{|c|}{ Pomares / CA } \\
\hline & & & BPAl & BPA2 & BPA3 & $\mathrm{PCl}$ & $\mathrm{PC2}$ & $\mathrm{PC3}$ \\
\hline \multirow[t]{9}{*}{ 2005/06 } & \multirow[t]{2}{*}{ Pragas } & Inseticida & 3 & -1 & -1 & -3 & -3 & -1 \\
\hline & & Monitoramento & 3 & 3 & 3 & -3 & -3 & 3 \\
\hline & \multirow{4}{*}{$\begin{array}{l}\text { Plantas } \\
\text { invasoras }\end{array}$} & Herbicida & 3 & 1 & 1 & -1 & -1 & 1 \\
\hline & & Manejo & -1 & 3 & 1 & -1 & -3 & -1 \\
\hline & & Diversidade & 1 & 3 & 3 & -1 & -1 & 1 \\
\hline & & Distribuição & 3 & 3 & 1 & -3 & -1 & 3 \\
\hline & \multirow{3}{*}{$\begin{array}{l}\text { Inimigos } \\
\text { naturais }\end{array}$} & Diversidade & 3 & 1 & 1 & 1 & 1 & 1 \\
\hline & & Densidade & -3 & -1 & -1 & -1 & -3 & -3 \\
\hline & & Seletividade $^{1}$ & -1 & -5 & -3 & -6 & -9 & -9 \\
\hline \multicolumn{3}{|c|}{ Subtotal dos coeficientes de alteração 2005/06 } & 11 & 7 & 3 & -18 & -23 & -5 \\
\hline \multirow[t]{9}{*}{ 2006/07 } & \multirow[t]{2}{*}{ Pragas } & Inseticida & 3 & 3 & 1 & -1 & -1 & -1 \\
\hline & & Monitoramento & 3 & 3 & 3 & -3 & -3 & 3 \\
\hline & \multirow{4}{*}{$\begin{array}{l}\text { Plantas } \\
\text { invasoras }\end{array}$} & Herbicida & 3 & 1 & -1 & -3 & 1 & 1 \\
\hline & & Manejo & -1 & 3 & -1 & -1 & -3 & -1 \\
\hline & & Diversidade & 1 & 3 & 3 & -1 & -1 & 1 \\
\hline & & Distribuição & 3 & 3 & 1 & 1 & -1 & 3 \\
\hline & \multirow{3}{*}{$\begin{array}{l}\text { Inimigos } \\
\text { naturais }\end{array}$} & Diversidade & 3 & 3 & 3 & 1 & 1 & 1 \\
\hline & & Densidade & -1 & 1 & 1 & 3 & -3 & 1 \\
\hline & & Seletividade ${ }^{1}$ & -1 & -1 & -6 & -3 & $-2^{2}$ & -9 \\
\hline \multicolumn{3}{|c|}{ Subtotal dos coeficientes de alteração 2006/07 } & 13 & 19 & 4 & -7 & -12 & -1 \\
\hline \multicolumn{3}{|l|}{ Total } & 24 & 26 & 9 & -25 & -35 & -6 \\
\hline \multicolumn{3}{|c|}{ Conceito de impacto ambiental ${ }^{3}$} & MD & MD & CI & MA & GA & $\mathrm{CI}$ \\
\hline
\end{tabular}

${ }^{1}$ Os valores de seletividade de inseticidas estão somados, devido ao número de aplicações realizadas, os quais podem ser verificadas na Tabela 1.

${ }^{2}$ Não foram considerados os dados referentes ao ingrediente ativo Lambdacyhalothrin por falta de informações quanto à seletividade para os inimigos naturais.

${ }^{3}$ Grande diminuição da possibilidade de impacto ambiental (GD), Moderada diminuição da possibilidade de impacto ambiental (MD), Componentes inalterados quanto ao impacto ambiental (CI), Moderado aumento da possibilidade de impacto ambiental (MA) e Grande aumento da possibilidade de impacto ambiental (GA)

TABELA 5 - Inimigos naturais (I.N.) e afídeos coletados em pomares de pessegueiros 'Chimarrita' conduzidos pelo sistema de Boas Práticas Agrícolas (BPA) e Produção Convencional (PC).Araucária-PR. Julho de 2005 a setembro de 2006.

\begin{tabular}{|c|c|c|c|c|c|c|c|}
\hline \multirow{2}{*}{ Pomares } & \multicolumn{5}{|c|}{ Número de inimigos naturais / Família } & \multirow{2}{*}{$\begin{array}{c}\text { Porcentagem } \\
\text { de I.N. }\end{array}$} & \multirow{2}{*}{$\begin{array}{l}\text { Número de } \\
\text { afideos }\end{array}$} \\
\hline & Syrphidae & Coccinellidae & Chrysopidae & Hymenoptera & Total & & \\
\hline BPA1 & $39 \mathrm{a}$ & $42 \mathrm{a}$ & $3 a$ & $58 \mathrm{a}$ & 142 & $11,0 \mathrm{a}$ & $1785 a b c$ \\
\hline BPA2 & $59 \mathrm{a}$ & $80 \mathrm{a}$ & $6 \mathrm{a}$ & 111a & 256 & $20,0 \mathrm{a}$ & $3660 \mathrm{bc}$ \\
\hline BPA3 & $97 \mathrm{a}$ & $72 \mathrm{a}$ & $8 a$ & $123 a$ & 300 & $22,9 \mathrm{a}$ & $1147 a$ \\
\hline $\mathrm{PCl}$ & $83 a$ & $104 a$ & $9 a$ & $109 \mathrm{a}$ & 305 & $23,5 \mathrm{a}$ & $4326 \mathrm{c}$ \\
\hline $\mathrm{PC} 2$ & $55 \mathrm{a}$ & $21 a$ & $4 a$ & $32 \mathrm{a}$ & 112 & $8,6 \mathrm{a}$ & $1139 a b c$ \\
\hline $\mathrm{PC} 3$ & $38 \mathrm{a}$ & $31 \mathrm{a}$ & $7 \mathrm{a}$ & $105 \mathrm{a}$ & 181 & $14,0 \mathrm{a}$ & $999 \mathrm{ab}$ \\
\hline Total & 371 & 350 & 37 & 538 & 1296 & 100,0 & 13056 \\
\hline
\end{tabular}

TABELA 4 - Plantas invasoras presentes em pomares de pessegueiros 'Chimarrita' conduzidos pelo sistema de Boas Práticas Agrícolas (BPA) e Produção Convencional (PC).Araucária- PR. Julho de 2005 a setembro de 2006.

\begin{tabular}{|c|c|c|c|c|c|c|c|c|}
\hline \multirow{2}{*}{ Família } & \multirow{2}{*}{ Nome cientifico } & \multirow{2}{*}{ Nome comum } & \multicolumn{6}{|c|}{ Pomares com presença de plantas invasoras } \\
\hline & & & BPAl & $\mathrm{BPA2}$ & $\mathrm{BPA3}$ & $\mathrm{PCl}$ & & $\mathrm{PC} 3$ \\
\hline \multirow{8}{*}{ Asteraceae } & Galinsoga parviffora Cav. & Picão-branco & & & $\mathrm{x}$ & $\mathrm{x}$ & & \\
\hline & Artemisia verlotorum Lamote & Losna & & & $x$ & & & \\
\hline & $\begin{array}{l}\text { Bidens pilosa } \mathrm{L} . \\
\text { Hypochoeris brasiliensis (Less). }\end{array}$ & $\begin{array}{l}\text { Picão-preto } \\
\text { Almeirão do }\end{array}$ & & & $x$ & $x$ & & \\
\hline & Griseb & campo & $x$ & & $x$ & & $x$ & ${ }^{A}$ \\
\hline & Raphanus sativus L. var. oleiferus & Nabo forrageiro & & $x$ & & & & \\
\hline & Sonchus oleraceus L. & Serralha & & $\mathrm{x}$ & $\mathrm{x}$ & & $x$ & $\mathrm{x}$ \\
\hline & Soliva pterosperma (Juss.) Less. & Roseta & & & $x$ & & & \\
\hline & Taraxacum offcinale Weber & Dente-de-leão & $x$ & & & & $x$ & $\mathrm{x}$ \\
\hline Commelinaceae & Commelina benghalensis $\mathrm{L}$. & Trapoeraba & & & & & & $\mathrm{x}$ \\
\hline \multirow[t]{3}{*}{ Cruciferae } & Brassica rapa $\mathrm{L}$. & Mostarda & & $\mathrm{x}$ & & & & \\
\hline & Raphanus raphanistrum $\mathrm{L}$. & Nabiça & & $x$ & $x$ & & & \\
\hline & Raphanus sativus L. var. oleiferus & Nabo for & $x$ & & & & & \\
\hline \multirow[t]{3}{*}{ Gramineae } & Avenis strigosa Schreb & Aveia-preta & & $x$ & & & & \\
\hline & Brachiaria mutica (Forsk.) Stapf & Capim-angola & & $x$ & & & $x$ & \\
\hline & Brachiaria plantaginea (Link) Hitchc. & Capim-marmelada & & $x$ & $x$ & & & $\mathrm{x}$ \\
\hline \multirow[t]{2}{*}{ Leguminosae } & Vicia sativa $\mathrm{L}$. & Ervilhac & $\mathrm{x}$ & $x$ & & & & \\
\hline & Vicia villosa Roth cv. An & Ervilhac & $x$ & $x$ & & $x$ & & \\
\hline Malvaceae & Sida rho & Guanxuma & & & & $x$ & $x$ & \\
\hline \multirow[t]{2}{*}{ Oxalidaceae } & Oxalis co & Azedinha & & & $x$ & & & \\
\hline & Oxalis latifolia Kunth & Trevo & & & $x$ & & & \\
\hline Plantaginaceae & Plantago tomentosa Lam. & Tanchagem & & $x$ & $x$ & & & \\
\hline Polygonaceae & Rumex obtusifolius L. & Lingua-de-vaca & & & & & & $x$ \\
\hline Pontederiaceae & Heteranthera limosa (Sv.) Willd. & Língua-de-cervo & $x$ & & & & & \\
\hline Solanaceae & Solanum americanum Mill. & Maria-pretinha & & & & $\mathrm{x}$ & & \\
\hline
\end{tabular}

\section{CONCLUSÃO}

Nas condições em que foi realizado o presente trabalho,pode-se concluir que o número de inimigos naturais não foi diferente entre os sistemas de produção.

\section{REFERÊNCIAS}

ACTA - Association de Coordination Technique Agricole. Index phytosanitaire. Paris : Acta Service Lutte Antiparasitaire, 1993. 529 p.

ACTA - Association de Coordination Technique Agricole. Index phytosanitaire. Paris : Acta Service Lutte Antiparasitaire, 2006. $824 p$.

ACTA/OILB. Controles seuils et indications pour la lutte. Paris : Pommier III, 1974. 75p.

AGNELLO, A.; KOVACH, J.; NYROP, J.; REISSIG, H.; WILCOX, $\mathrm{W}$. Aguide for sampling and managing major apple puts in New York State. New York: State Integrated Pest Management Program, 1993. 
ALTIERI, M. A; SILVA, E. N.; NICHOLLS, C. I. O papel da biodiversidade no manejo de pragas. Ribeirão Preto: Holos, 2003. 226p.

ALTIERI, M; NICHOLLS, C; FRITZ, M. A. Manage insects on your farm: a guide to ecological strategies. Beltsville: Sustainable Agriculture network, 2005.130p.

BARBIN, D. Componentes de variância: teoria e aplicações. Piracicaba: FEALQ, 2004. 120p.

CAMPANHOLA, C.; LUIZ, A. J. B.; LUCCHIARI JÚNIOR, A. O problema ambiental no Brasil: agricultura. In: ROMERO, A. R.; REYDON, B. P.; LEONARDI, M. L.A. Economia do meio ambiente: teoria, políticas e gestão de espaços regionais. Campinas: UNICAMP, 1997. p.265-281.

DELABAYS, N.; LINDER, C.; VIRET, O. Index phytosanitaire pour l'arboriculture 2003 publié avec le soutien de l'Office federal de l'environnement des forêsts et du paysage. Revue Suisse de Viticulture, Arboriculture, Hoticulture, Paris, v.35, n.1, 2003 a. Suplemento.

DELABAYS, N.; LINDER, C.; VIRET, O. Le guide arbo de changes 2003-2005. Revue Suisse de Viticulture, Arboriculture, Hoticulture, Paris, v.35, n.1, 2003b. Suplemento.

FACHINELLO, J. C.; COUTINHO, E. F.; MARODIN, G.; B.; BOTTON, M.; MAY DE MYO, L. L. Normas técnicas e documentos de acompanhamento da produção integrada de pêssego. Pelotas: Universidade Federal de Pelotas, 2003. 92 p.

FACHINELLO, J. C.; HERTER, F.G. Normas para produção integrada de frutas de caroço (PIFC). Pelotas: Embrapa/ CPACT, 2001.92p

FADINI, M. A. M.; LOUSADA, J. C. N. Impactos ambientais da agricultura convencional. Informe Agropecuário, Belo Horizonte, v.22, n.213, p.24-29, 2001.

HULL, A. L.; FELLAND, C. M.; BIDDINGER, D. J. Insecticide and acaricide evaluations for management of apple, pear, and peach pests and tolerance by natural enemies. Biglerville: Departament of Entomology Penstate University Fruit Research an Extension Centre, 1994. 28p.
KOVACH, J.; PETZOLDT, C.; DEGNI, J.; TETTE, J. A method to measure environmental impact of pesticides. New York's Food and Life Sciences Bulletin, New York, n.139, 8p., 1992.

LETOURNEAU, D. K; ALTIERI, M. A. Abundance patterns of the predator Orius tristicolor (Hemiptera: Anthocoridae) and its prey, Frankliniella occidentalis (Thysanoptera: Thripidae): habitat attraction in polyculturus versus monocultures. Environmental Entomology, Baltimore, v.122, p.1464-1469, 1983.

MARANGONI, B.; TAGLIAVINI, M.; SCUDELARI, D. La gestione de suelo I'rrigazione e la fertilizzazione del pesco. In: La Peschiciltura Veronese alla Soglia de 2000. Verona: Atti del convegno, 1995. p.273-294.

PINEDA, A. S. V. Se puede optimizar el Monitoreo de $C y d i a$ Molesta (Busck) (Lepidoptera: Tortricidae) com Tranpas de Feromona? 2005. Monografia (Projeto de Conclusão de Curso de Engenheiro Agrônomo) - Universidade de Chile, Santiago, 2005.

PRICE, P. W. Interactions among three trophic levels: influence of plants on interaction between insect herbivores and natural enemies. Annual Review Ecology Sistematcs, Palo Alto, n.11, p.41-65, 1980.

RODRIGUES, G. S.; CAMPANHOLA C.; KITAMURA, P. C. Avaliação de impacto ambiental da inovação tecnológica agropecuária: um sistema de avaliação para o contexto institucional de P\&D. Cadernos de Ciência \& Tecnologia, Brasília, v.19, n.3, p.349-375, 2002.

SIMEPAR. [on line]. Disponível em $<$ http://www.simepar.br/>. Acesso em: 30 out. 2006.

SPV - Service de la Protection des Vegetaux. Les actions secondaires dês produits phytosanitaires. $5^{\text {th }} \mathrm{ed}$. Toulouse: Union dês industries de la protection des plantes, 1983.

TITI, A.E.L.; BOLLER, E.F.; GENDRIER, J.P. Producción integrada: principios y directrices técnicas. Dijon: IOBC/WPRS, 1995.22p. (Bulletin, 18).

UNION INTERNATIONALE DES SCIENCES BIOLOGIQUES. Action des pesticides sur la faune auxiliaire des arbes frutiers. Paris, 1986. 97p. (Bulletin Strop) 